This is the post peer-review accepted manuscript of:

M. Magno, L. Spadaro, J. Singh and L. Benini, "Kinetic energy harvesting: Toward autonomous wearable sensing for Internet of Things," 2016 International Symposium on Power Electronics, Electrical Drives, Automation and Motion (SPEEDAM), Anacapri, 2016, pp. 248-254.

The published version is available online at:

https://doi.org/10.1109/SPEEDAM.2016.7525995

C2016 IEEE. Personal use of this material is permitted. Permission from IEEE must be obtained for all other uses, in any current or future media, including reprinting/republishing this material for advertising or promotional purposes, creating new collective works, for resale or redistribution to servers or lists, or reuse of any copyrighted component of this work in other works. 


\section{Kinetic energy harvesting: toward autonomous wearable sensing for Internet of Things}

\author{
Michele Magno*, Lorenzo Spadaro \\ ${ }^{\circ}$ DEI- University of Bologna \\ Bologna, Italy
}

\author{
Jaskirat Singh, Luca Benini ${ }^{\circ}$ \\ *D-ITET. ETH Zurich, \\ Zurich, Switzerland
}

\begin{abstract}
Power supply availability is a limiting factor in wearable devices whose form factor constrains battery size. Wearable harvesters that collect energy from the environment are a promising technology to achieve the long-life goal for truly wearable devices. However, most of the mature energy harvesting approaches usually imply an outdoor setting, using solar panels, wind turbines or high-frequency vibration as energy sources. In particular, most kinetic harvesters are not effective with human motion frequencies and mobility patterns. In this paper, we evaluate and integrate a highly-efficient kinetic harvester circuit to power autonomous wearable devices, exploiting the energy gathered from human motion. For this purpose, a Kinetron Micro Generator System 26.4 (MSG) has been characterized, conversion circuits have been designed and evaluated using off-the-shelf components. The implemented kinetic harvesting system successfully manages to harvest energy from human movements and stores it in small supercapacitors or batteries. Experimental results show that it is possible to harvest up to $280 \mu \mathrm{J}$ from a single small human movement and up to $1.1 \mathrm{~J}$ per-day using a passive rectifier. This results in up to $180 \mu \mathrm{J}$ for single burst of energy and $0.7 \mathrm{~J}$ per-day stored energy in a battery following a direct current conversion. These are extremely promising results for small wearable kinetic harvesting, and demonstrate that this new generation of kinetic energy harvesters can be instrumental in the design of many self-sustainable wearable devices.
\end{abstract}

Keywords- Energy Harvesting, Kinetic energy harvseting, low power circuits, Wearable devices, energy efficiency,

\section{INTRODUCTION}

The Internet of Things (IoT) is a rapidly emerging application space, poised to become the largest electronics market for the semiconductor industry [1]. A fast growing class of IoT devices is that of wearables, where network nodes are tightly coupled with the human body [2]. Low power consumption is even more crucial in wearable systems due to the limited energy availability of the battery, which has stringent weight and size constraints. In fact, although integrated circuits have significantly improved their energy efficiency thanks to Moore's law, battery technology is not making similar strides. Thus, low power design alone is not enough to make these devices really wearable. The battery lifetime of a truly wearable design has to be in the order of months if not years instead of daily recharges common to contemporary smart phones and wearables.

Harvesting energy from environmental sources such as light and human movements has been recognized as the most promising approach to supply wearable and IoT devices [3].
Even small movements during daily activities of humans produce a significant amount of energy which could be harvested [1]. In recent years, many approaches tried to insert generators in shoes' soles to generate energy during the walking activity [3]-[5]. Other approaches focused on embedding generators in backpacks to charge smartphones or mobile device. However, smaller form factor kinetic energy harvesters are needed in IoT applications and especially in wearable devices. To achieve this goal the whole energy harvester including the electromechanical generator should satisfy weight (below few grams) and size (i.e. $40 \mathrm{~mm} \times 40 \mathrm{~mm}$ so that it can be placed under a smart watch) requirements and should generate at least few hundreds of micro-Watts [6], which could suffice for many applications [7]. Since small generators harvest a limited amount of energy, it is important to transfer this energy from the generator to an energy buffer (i.e. capacitors or batteries) as efficiently as possible. Harvesting meaningful amounts of energy from such constrained harvesters is a challenge that requires a significant amount of research and development effort.

In this work, we present a novel kinetic energy harvesting system comprised by a micro-generator and the related conversion electronics which satisfies the stringent requirements of size, weight, power harvested and stored. We use a miniaturized kinetic generator which measures only $26.4 \mathrm{~mm}$ in diameter, is $4.3 \mathrm{~mm}$ of height, weighs 4.5 grams, generating up to $3 \mathrm{~mW}$ of power and around $530 \mu \mathrm{J}$ of energy from small movements. Following an in-depth characterization of the kinetic generator aimed to determine the conditions which maximize its power output, we evaluated possible harvesting architectures to achieve the most energy efficient solution that maximizes the energy usable by the load. The harvesting architectures include the rectifier from the $\mathrm{AC}$ output of the micro generator, and DC-DC converters to store the energy in a supercapacitor or a Li-Ion battery under different application conditions. The proposed solution achieves up to $68 \%$ conversion efficiency when the circuits goes out of the cold start phase. The architecture of the conversion circuit minimizes the overall power consumption and has been developed by using off-the-shelf (COTS) components and state of the art integrated circuits. The whole harvester device measures $40 \mathrm{~mm}$ x $40 \mathrm{~mm}$ with a weight of 5 grams and can be plugged inside many small sized wearable devices and allows to store up to $180 \mu \mathrm{J}$ from small movements.

The reminder of the paper is organized as follows: Section II briefly describes the state of the art in the field. Section III details 
the architecture of the system from hardware and software point of view. In Section IV results of measurements performed on the system are presented, and conclusions are drawn in Section V.

\section{RELATED WORK}

Over the last decade, wearable technology has received increasing attention from industrial as well as academic communities. This manifests itself by the ever-increasing research and development efforts in this field. Many commercial wearable devices became successful products in the wellness, sport domain and there are many applications that interface directly with a mobile phone [1]. Achieving a self-powered system through the use of energy harvesters $(\mathrm{EH})$, that capture energy from the environment, combined with various storage devices such as batteries (commonly LiPo, rechargeable) and super-capacitors is a particularly interesting and mature approach [8]-[14]. Since wearable devices are expected to be mostly used on the human body, the most commonly available ambient energy sources are photovoltaic, thermal or vibrational energy [15], [16].

Oscillating rotational generators originated from wristwatch companies. The Japanese company Seiko presented the Automatic Generating System (AGS) in 1986. This self-winding mechanism was used on wristwatches under the Kinetic brand name. The design consisted of a rotating pendulum mass, a gear box train (ratio 1:100), and a small permanent magnet generator. Due to wrist position changes, one oscillation from the pendulum-mass produced 100 rotations on the generator. According to Paradiso and Starner[5], 5-10 $\mu \mathrm{W}$ of power was estimated to be produced when worn, and up to $1 \mathrm{~mW}$ could be obtained when forcibly shaken. Swiss company ETA later introduced the Autoquartz with a different approach. The pendulum mass wound a spring connected to a small generator using a gear box train. Once the spring was fully wound, it unwound making the generator rotate at $5-15 \mathrm{krpm}$ for a short time (50 ms), generating more than $15 \mathrm{~V}$ and $6 \mathrm{~mA}(90 \mathrm{~mW})$ [5]. In this work, we use a novel micro generator system (MGS 26.4) developed by Kinetron [17] which unlike the previous generator, directly provides the electrical AC output of up to $3 \mathrm{~mW}$ and can, then be used as source for a conversion and storage circuit.

In [18], Mitcheson concludes that although it is a very popular choice in mechanical watch industry, the amount of energy harvested by kinetic energy converters is orders of magnitude less than what can be harvested from thermal and solar energy harvesters. However in [2] the authors present an interesting estimation of harvested energy using acceleration traces, which shows that it is possible to harvest micro-Watts of energy from human movement and this energy is sufficient for many application scenarios in the IoT domain. The authors in [2] relied on a model and did not consider the effects of the conversion circuit. As a result their results are a bit optimistic. In this work, we present the complete harvesting circuit with a commercial state-of-the-art micro-generator and present in-field measurements which confirms that kinetic energy harvesting produces comparable energy to other sources and can be a viable option for IoT applications.

A solution to collect energy using a thermal generator attached to the human body is presented in [19]. Solar energy harvesting is a mature technology and guarantees much higher values of energy [8]. However indoor light harvesting, especially for wearable devices as in our approach, is still very challenging and novel. In [7] an ultra-low-power multi-sensor wearable-device has been equipped with an indoor solar harvester. The authors measured the harvested energy in indoor conditions during several days and demonstrated the wearable device can be self-sustainable. Also in [7], the authors show that it is possible to generate an average of $0.6 \mathrm{~J}$ per day from eight small form factor solar panels placed in the wristband and using highly efficient conversion circuits. In this paper we demonstrate that this amount of harvested energy can be achieved and in specific conditions (i.e when running) even exceeded by kinetic harvesting. This is an important result, as it shows that potentially kinetic energy can be used instead of solar energy, or it be can be coupled in a hybrid system combining both kinetic and solar energy harvesting for increased flexibility.

Piezoelectric generators for energy harvesting of human movements have also been studied in literature. Shenck and Paradiso [4] exploited the energy released during the walking process to scavenge energy from piezoelectric materials mounted at the sole of the shoes. However piezoelectric generators only work when the resonant frequency is stable and quite high (more than $40 \mathrm{~Hz}$ ), for this reason they are considered unsuitable for wearable applications.

Finally, in contrast to many other approaches[2][3][5] [8]which are only working models, simulations or a single component of the harvesting circuit (i.e. only the generator, the AC-DC conversion, DC-DC or the storages), our focus is to evaluate the kinetic harvesting as a whole system and design it to achieve the maximum performance in terms of efficiency and energy harvested as well as test the system in real application scenarios.

\section{KinETIC HARVESTER ARCHITECTURE}

Energy harvesting is defined as the process by which energy is collected from the environment employing a generator that transforms the input energy into electrical energy to power autonomous electronic devices. Usually, the main components of harvesting circuits are:

Energy transducer (also known as harvester generator): is the device that converts some available ambient/movement energy into electrical energy. The environmental energy sources can be grouped by: thermal (thermoelectric cells), light (photovoltaic cells), RF (rectifying antennas) and mechanical (piezoelectric, kinetic). In this work we focus only on kinetic energy sources.

Converter: the electrical output of the energy transducer can not always be directly used as it can be in a different voltage or current range (i.e. too low voltage or too high current). Therefore, in most cases, it is necessary to convert the electrical output with an AC-DC converter (full bridge rectifier, voltage doubler) which can rectify the current, and/or using a DC-DC converter to achieve the desirable values of current and/or voltage.

Electronic load: this is the consumer of the energy. It is an electronic device connected to the end of the chain and typically has different power consumption modes. In most IoT applications, the device is frequently in a low-power 
consumption mode and operates in active mode only during a limited time.

Storage element: an optional battery or capacitor is used to store the harvested energy to use it in the future or as a buffer to stabilize the intake energy. In some cases, the electronic load can be completely switched off and therefore the battery can be used to exploit all of the collected energy.

Power management: a circuit (often part of an integrated circuit) designed to optimize effective use of the harvested energy by the electronic load. Usually, this is one of the few components to remain active even when the rest of the system is in sleep mode since it controls the different operation modes of the system.

Fig. 1 shows the block diagram of the kinetic harvesting system considered in this work. It consists of the micro generator that converts kinetic energy from movements into electrical energy, an AC-DC converter to convert the AC output of the generator to a DC current, and finally a DC-DC converter to supply the load and energy storage. The whole device is holistically designed to be ultra-low power and to work with the intermittent and low energy generated by the kinetic microgenerator. Thus, the design uses as much as possible passive components (i.e. the AC-DC converter) and ultra-low power integrated circuits (i.e. the DC-DC and storage management) to keep the quiescent and active energy consumption very low. In the following subsection we describe the individual subsystems.

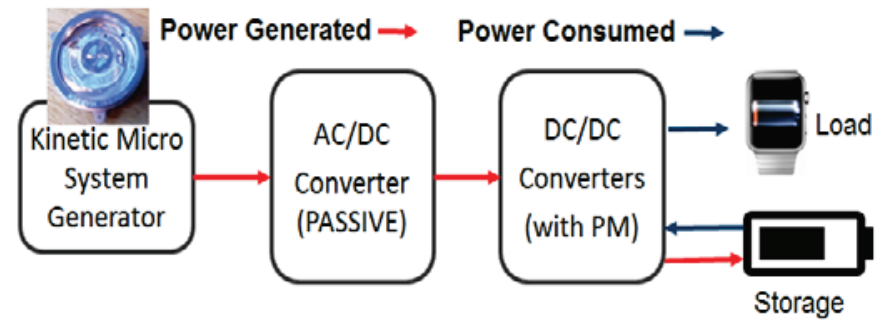

Fig. 1. Kineti Energy Harvesting Block Architecture.

\section{A. Kineri Micro system Generator}

The principle behind kinetic energy harvesting is the displacement of a moving part or the mechanical deformation of some structure. This displacement or deformation can be converted to electrical energy in three different ways: inductive, electrostatic and piezoelectric conversion. Kinetic energy can be transformed into electrical energy with inertial and noninertial transducers. The former are based on a spring-mass system where the mass vibration or displacement can be exploited. For non-inertial converters, an external element applies a pressure that is transformed into elastic energy, causing a deformation that is converted to electrical energy by the converter.

The selected kinetic generator is an inductive electromagnetic one. Electromagnetic generators are based on the induced voltage in a coil when a magnet moves relative to it. This is produced by the changing in the magnetic flux as described by the Faraday's Law of induction:

$$
|E|=\left|\frac{d \phi_{B}}{d t}\right|
$$

Where $|E|$ is the magnitude of the electromotive force (EMF) in volts and $\phi_{B}$ is the magnetic flux. This change is either due to having a fixed magnet and a moving coil, or the opposite, a fixed coil and moving magnet. For a coil, the EMF depends on the number of coil turns, the strength of the magnetic flux and the rate of change of the magnetic flux.

An overview of the output power of different kinetic converters is given in the following table:

TABLE I.

\begin{tabular}{|l|l|l|l|}
\hline \multicolumn{1}{|c|}{$\begin{array}{c}\text { Design } \\
\text { Author }\end{array}$} & $\begin{array}{l}\text { Mechanical } \\
\text { excitation }\end{array}$ & $\begin{array}{l}\text { Output } \\
\text { power }\end{array}$ & Dimensions \\
\hline Roundy et al. [20] & $\begin{array}{l}\mathrm{f}=40 \mathrm{~Hz} \\
\mathrm{a}=2.25 \mathrm{~ms}^{-2}\end{array}$ & $\begin{array}{l}1.7 \mathrm{~mW} \\
@ 12 \mathrm{~V}\end{array}$ & $4.8 \mathrm{~cm}^{3}$ \\
\hline Hu et al. [21] & $\begin{array}{l}\mathrm{f}=50 \mathrm{~Hz} \\
\mathrm{a}=1 \mathrm{~ms}^{-2}\end{array}$ & $\begin{array}{l}256 \mu \mathrm{W} \\
@ 18.5 \mathrm{~V}\end{array}$ & $\mathrm{n} / \mathrm{a}$ \\
\hline Jeon et al. [22] & $\begin{array}{l}\mathrm{f}=13900 \mathrm{~Hz} \\
\mathrm{a}=107 \mathrm{~ms}^{-2}\end{array}$ & $\begin{array}{l}1 \mu \mathrm{W} @ \\
3 \mathrm{~V}\end{array}$ & $0.027 \mathrm{~mm}^{3}$ \\
\hline Liu et al. [23] & $\begin{array}{l}\mathrm{f}=229 \mathrm{~Hz} \\
\mathrm{a}=4.9 \mathrm{~ms}^{-2}\end{array}$ & $\begin{array}{l}3.98 \mu \mathrm{W} \\
@, 4 \mathrm{~V}\end{array}$ & $1 \mathrm{~mm}^{3}$ \\
\hline Renaud et al. [24] & $\begin{array}{l}\mathrm{f}=1.8 \mathrm{kHz}_{\mathrm{m}} \\
\mathrm{a}=1.18 \mathrm{~ms}^{-2}\end{array}$ & $40 \mu \mathrm{W}$ & $7.5 \mathrm{~mm}^{3}$ \\
\hline
\end{tabular}

In this work the Micro Generator System 26.4 (MSG26.4) [17] has been exploited as electromagnetic harvester due to its small form factor (can be worn on the wrist). It is equipped with a 4.5 gram oscillating weight with a gear which drives two mechanical rectifiers. One of the mechanical converters contains a spring barrel that brings the torque to its gear which, finally, drives the rotor of the micro generator (MG4.0). The MG4.0 consist of a 14-pole magnet and claw pole stator that encloses a coil with 1140 windings at a resistance of $320 \Omega$. Each complete rotation of the magnet in the stator induces an $\mathrm{AC}$ voltage with 7 sinusoidal waves. The amplitude of the AC voltage is proportional to the speed of the turning magnet.

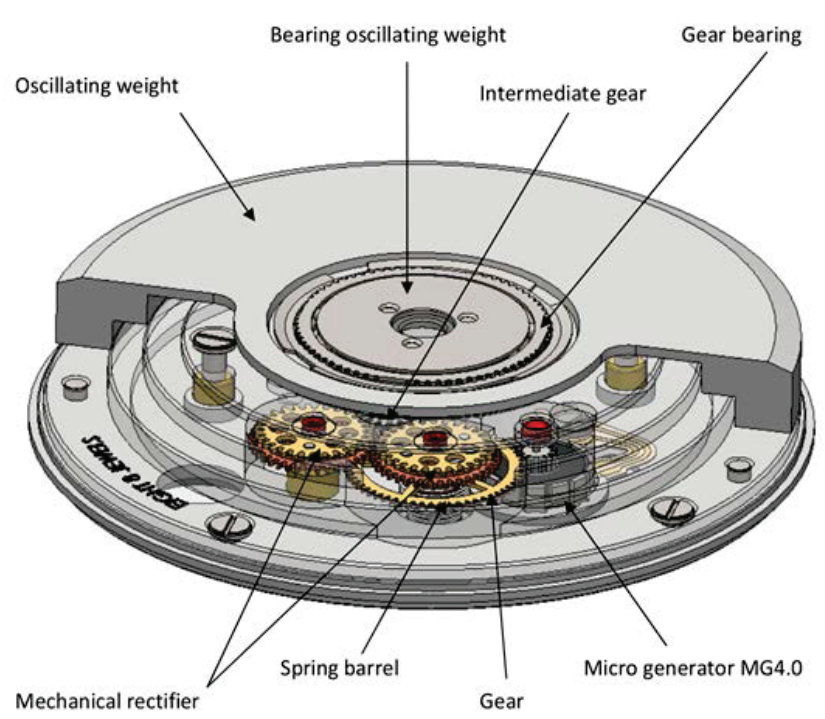

Fig. 2. MSG26.4 harvester main components displayed [17] 
Fig. 3 shows the AC waveforms generated by the MSG during a half spin of the oscillating weight. A half spin was done inclining the harvester by $90^{\circ}$ and slowly letting the oscillating mass rotate without any initial force applied. This process allows us to evaluate the voltage, power and energy provided by the MSG.

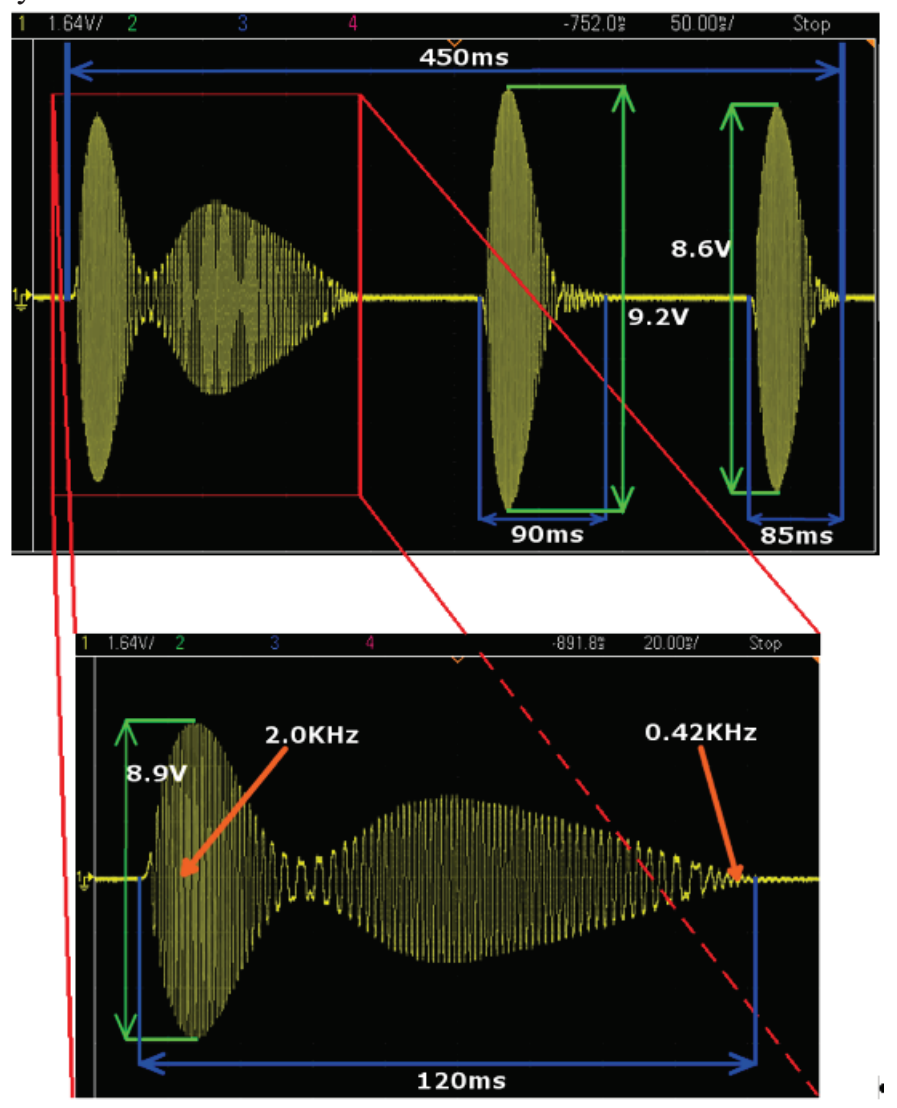

Fig. 3. Half-spin waveforms generated from the MSG. The half spin generates three bursts of energy. The figure below highlights only one burst.

To measure the total power, a discrete integration method has been used (Fig. 4) by matching the internal resistance of 320 $\mathrm{Ohm}$ of the generator. Each waveform was divided into 15 equal divisions for the first wave (which is longer) while the second and the third waves were divided into 10 equal intervals. After this, for each subdivision the peak voltage $V p$ was measured and, using the following formula, the power in that specific interval was calculated:

$$
P_{i}(R M S)=\frac{\left(V p_{i} / \sqrt{2}\right)^{2}}{R}
$$

Where $R$ is the value of the variable resistor used for the measurements (320Ohm). Finally, using the equation 2, the total power in the analysed waveform have been retrieved summing all the burst generated for single whole spin.

$$
P_{R M S}=\frac{1}{n} \sum_{n}^{N} P_{i}(R M S)
$$

where $n$ is the number of divisions. The power and energy was measured for a full spin of the oscillating weight by measuring the AC output voltage of the generator and then using the equation (2) and (3). To estimate the average value of power and energy we repeated the measurements for 100 times and have determined to be $3 \mathrm{~mW}$ and $539 \mathrm{uJ}$ for full spin rotation of the oscillating weight.

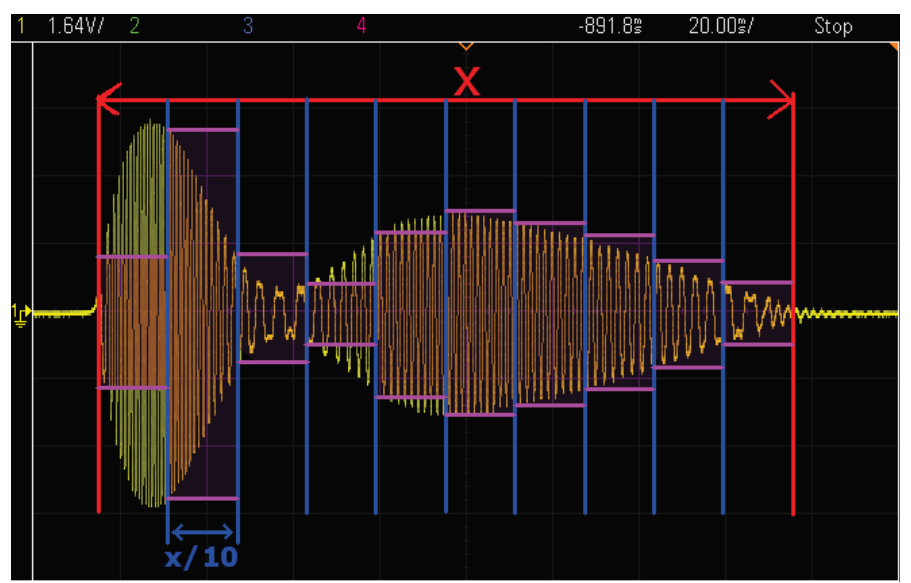

Fig. 4. General description of the discrete sum used for calculating the area of the waveform for power estimation

\section{B. AC-DC converters}

The energy harvester generates an AC output that needs to be converted to DC for embedded devices. In this study a passive AC-DC converter has been use. In order to evaluate the most energy efficient configuration between passive rectifiers we considered two options: full bridge (Fig. 5) and a double voltage (Fig. 6). For both the converters Schottky diodes (BAT51) with very low voltage drop of $250 \mathrm{mV}$, have been used to maximize the power conversion with minimal loses.

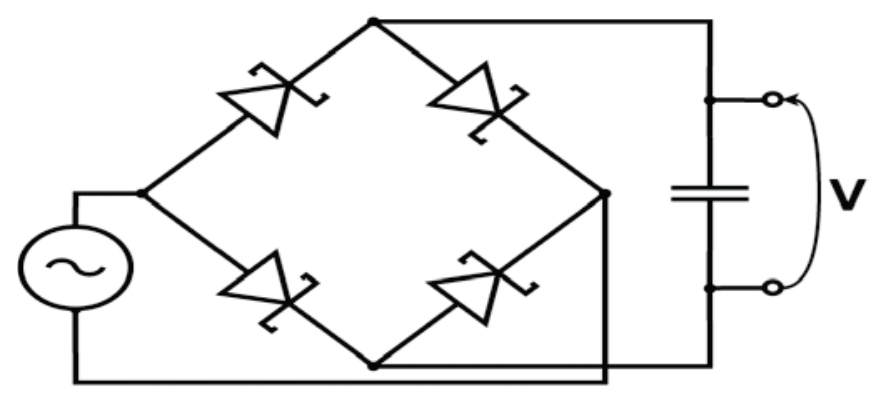

Fig. 5. : Full bridge rectifier circuit for energy measurements

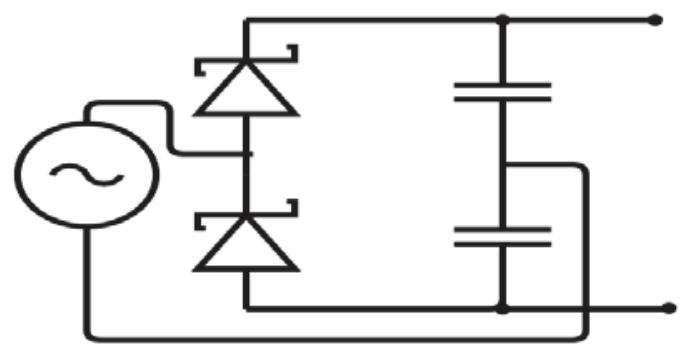

Fig. 6. : Doubler voltage rectifier circuit

Consequently, the energy accumulated into the rectifier capacitor (the series of the for the doubler voltage) have been 
calculated using the measured voltage of the capacitor and the following equation:

$$
W=\int_{t_{0}}^{t_{\max }} P(t) d t=\frac{1}{2} C\left[V\left(t_{\max }\right)^{2}-V\left(t_{0}\right)^{2}\right]
$$

Where $C$ is the value of the series of the output capacitance used; $V\left(t_{\max }\right)$ is the maximum voltage reached on the capacitor and $V\left(t_{0}\right)$ is the initial voltage on the capacitor. In the experimental results the comparison between the two architectures will be presented.

\section{DC-DC Converter}

A DC-DC converter is an electronic component that converts a direct current source at one voltage level to another DC level at a different voltage. This is especially important when batteries need to be recharged or the electronic load has maximum voltage restrictions (i.e. cannot exceed 3.3V). There are three different types of DC-DC converters: i) Buck converter which has a lower output level than its input, ii) Boost converter which has a higher output level than its input and iii) Buck-Boost converters that works when the input is both higher or lower than the output level. In this work we design and evaluate two different circuits: one with a Boost converter and a second utilizing a Buck-Boost converter designed by using commercial integrated circuits (IC) optimized for energy harvesting to reduce the quiescent current of the devices.

The Linear Technology LTC3129-1 has been used to implement a Buck-Boost DC-DC converter. This IC has high conversion efficiency at low power input that matches the kinetic generator, and includes a maximum power point control to ensure maximum power extraction from non-ideal power sources. Additionally the output voltage divider provides eight selectable fixed output voltages.

The Texas Instruments BQ25570 has been used to implement a boost charger. This IC is optimized for energy harvesters and contains a boost charger with a quiescent current of only $488 \mathrm{nA}$, a programmable step down regulator with an efficiency up to $93 \%$, and can be used to supply the load from the storage directly. This chip is specifically designed to extracts microwatts of power generated from numerous high output impedance DC sources (such as solar panels). Other traits include: battery overcharging control, programmable maximum power point tracking (MPPT) and highly integrated foot-print $(3.5 \mathrm{~mm} \times 3.5 \mathrm{~mm})$. Typical applications include solar chargers, TEG harvesting, environmental and structural health monitoring and wearable devices.

\section{EXPERIMENTAL RESULTS}

To evaluate the performance of the different harvester architectures several circuits have been implemented (Fig. 7). The developed prototype testbed has been evaluated experimentally with respect to energy harvesting performance and efficiency. Initial tests were made to evaluate individual blocks (AC-DC, DC-DC) and the entire amount of energy which can be harvested per day using the kinetic generator.

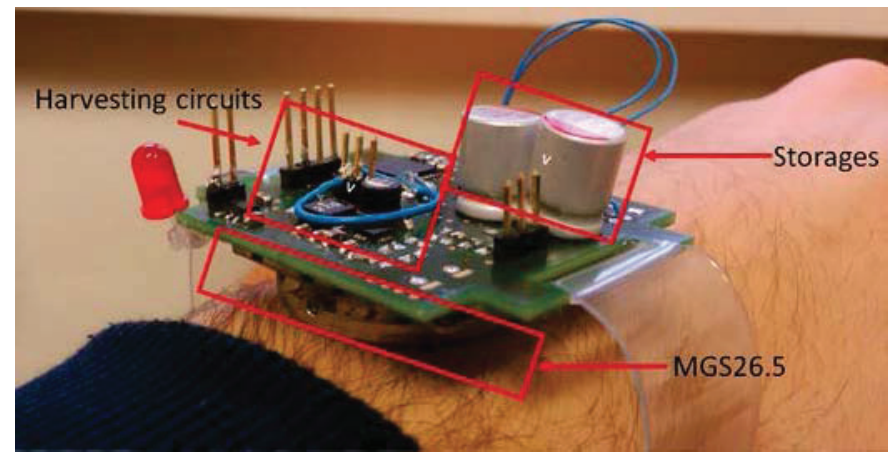

Fig. 7. Protototype of the kinetic energy harvesting used for the in-field measurmetns.

The first block that was evaluated was the passive ACDC converter using two proposed architectures. The following table summarizes the energy harvested from the kinetic harvesting following the AC-DC converter stage. With the energy of a single full spin, it is possible to estimate the energy per day. Kinetron datasheet [17], reports that human movements can potentially allow a full spin of the oscillating weight for 4000 times per day. For this reason an additional column is reported in the table which indicates the total energy expected to be harvested per day.

TABLE II. : AC-DC CONVERSION SUMMARY

\begin{tabular}{|l|c|c|c|c|}
\hline $\begin{array}{c}\text { AC/DC } \\
\text { type }\end{array}$ & $\begin{array}{c}\text { Half spin } \\
\text { energy } \\
{[\mathbf{J}]}\end{array}$ & $\begin{array}{c}\text { Full spin } \\
\text { energy } \\
{[\mathbf{J}]}\end{array}$ & $\begin{array}{c}\text { One day } \\
\text { average } \\
\text { energy } \\
{[\mathbf{J}]}\end{array}$ & $\begin{array}{c}\text { Efficiency } \\
{[\%]}\end{array}$ \\
\hline Full bridge & $119.0 \mathrm{E}-6$ & $230.0 \mathrm{E}-6$ & $920.0 \mathrm{E}-3$ & 42.14 \\
\hline $\begin{array}{l}\text { Voltage } \\
\text { doubler }\end{array}$ & $147.0 \mathrm{E}-6$ & $283.0 \mathrm{E}-6$ & $1.1 \mathrm{E}+0$ & 52.5 \\
\hline
\end{tabular}

The last column in Table II reports the efficiency of the converters as the difference of the average energy harvested when the AC output of the MSG is matched with the internal resistance as presented in section III $(539 \mu \mathrm{J})$ and the energy harvested after the rectifier. The table shows that the kinetic harvesting is able to generate a substantial amount of energy $(1.1 \mathrm{~J})$ per day, which would be sufficient for many applications. This is an impressive value especially when compared to the results presented in [7] where eight indoor wearable solar panels placed on the wrist band were reported to harvest around $0.6 \mathrm{~J}$ of energy per day. In our evaluation with the kinetic generator we could achieve almost $2 x$ of energy harvested when using the voltage doubler rectifier. It can be also observed the voltage doubler is the most efficient rectifier for the MSG with an efficiency of $52.5 \%$. These loses are due to the voltage drop across the diodes and cannot be removed for passive solutions. For the remainder of the evaluation only the more efficient voltage doubler has been used.

The second evaluation has been performed to evaluate the performance of the buck-boost and the boost converters. Fig. 8 shows the implemented circuit that uses the buck-boost LTC3119-1. In both setups the capacitors of the rectifier have been selected as $\mathrm{C}_{-} \mathrm{vd} 1=\mathrm{C}_{-} \mathrm{vd} 2=47 \mu \mathrm{F}$. These capacitors play both the role of rectifier capacitors and input capacitors of the buck-boost converter. For this evaluation a full spin of oscillating weight has been performed and the voltage accumulated on the output capacitance has been measured. The 
storage capacitor used is $\mathrm{C}_{\text {out }}$ of $470 \mu \mathrm{F}$. The system was evaluated in two different modes: i) cold start mode when the Cout starts from zero Volt, and normal mode where the $\mathrm{C}_{\text {out }}$ has an initial value of $2 \mathrm{~V}$. Using this setup the total energy harvested has been measured to be $100 \mu \mathrm{J}$ during the cold start mode and $140 \mu \mathrm{J}$ in normal mode. In terms of efficiency this results in an efficiency of $35.34 \%$ and $49 \%$ respectively. The losses are mostly due to the low efficiency of the commercial IC when dealing with low input power produced by kinetic generators.

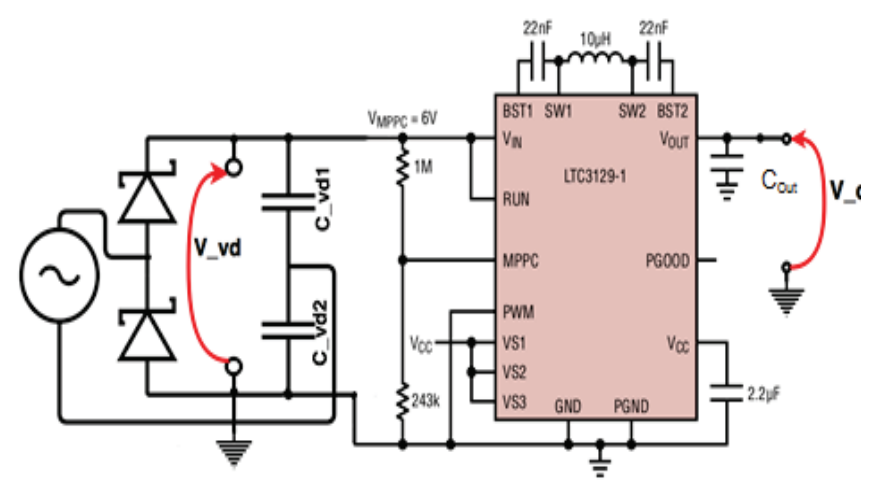

Fig. 8. LTC3129-1 energy harvesting circuit

Fig. 9 illustrates the second energy harvesting circuit that was designed. It uses the boost converter BQ25570 as its core IC, which employs 2 storages. One is used during the cold start mode $\left(\mathrm{C}_{\text {Store }}\right)$ and the other one $\left(\mathrm{C}_{\text {bat }}\right)$ can be a supercapacitor or a Li-Ion battery. To evaluate the efficiency of the whole harvester circuit both voltages of the capacitors has been measured. This IC is optimized for energy harvesting and provides an integrated power management to boost up the energy efficiency especially after the cold start mode. Moreover the IC integrates a cold start mode when the $\mathrm{V}_{\text {store }}$ is below 1.7V (Fig. 10). Unfortunately this phase is characterized by a very low conversion efficiency, however this is necessary to allow the IC to work in normal mode.

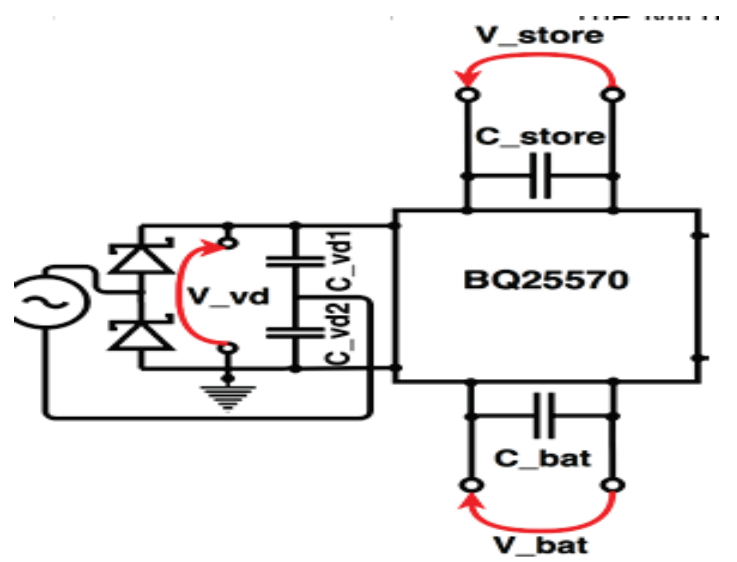

Fig. 9. BQ25570 energy harvesting circuit

As expected we observed that the efficiency in cold start mode is extremely low (less than 10\%) and therefore the behavior of the component has been studied outside of this state by forcing an initial voltage on the capacitors (Fig. 10). During the cold start mode in figure the oscillating weight has been made spin continously, and it can be oserved that are needed 2.8 seconds, which corrisponds to around 15 full spin, to exit of the cold start mode. During the normal mode, the BQ25570 harvested an average energy of $180 \mathrm{uJ}$ for full spin with an average conversion efficiency of $68 \%$. From the experimental results it is clear that the energy havesting circuits with the boost converter allows better performance in an application scenario where the cold start cases are rare (i.e. with Li-Ion batteries) while the buck-boost converter harvesting circuit is the better choice in batteryless application where cold starts happen more often.

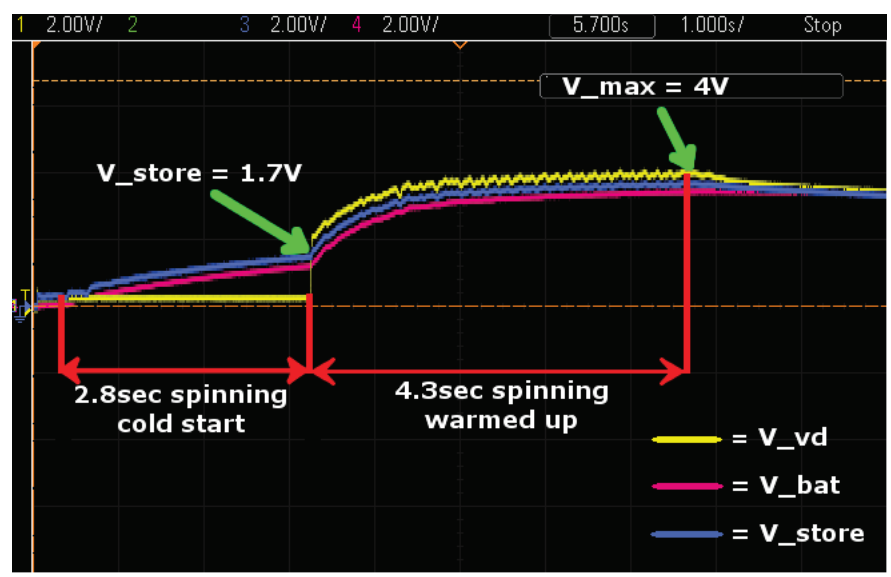

Fig. 10. Continous spinning of the kinetron to allow the exit of cold start

These results demonstrate that also when we use a DC-DC after the AC conversion the single full spin allow the storage of around $180 \mathrm{uJ}$. This means using the average spin number $(4000$ times) value for a normal day of human activities the estimated total stored and usable energy is $0.7 \mathrm{~J}$ per day which still exceeds the output of the eight solar panels as reported in [7].

\section{CONCLUSIONS}

The charaterization, evaluation, design and implementation of a wearable kinetic harvester that collects usable energy from human movements have been presented. The development of such as harvesting circuits represents a significant improvement over the current state-of-the-art and brings tangible values of accumulate energy using a novel micro generator and can be used in many wearable and IoT devices. The platform was designed for ultra-low power energy and optimized for the kinetic generator by evaluating different topologies of converters, Experimental results demonstrate that it is possible to harvest a considerable quantity of energy from small human movements. The estimated energy of the kinetic energy generator based on measurements indicate that up to $1.1 \mathrm{~J}$ can be generated per day. This is an impressive value and can enable self-suistanability of many wearable devices in several application domains. Future works will focus on long-term infield evaluation of the energy harvesting and even more optimized conversion circuits which can improve the overal efficiency. 


\section{ACKNOWLEDGMENT}

This work was supported by "Transient Computing Systems", a SNF project (200021 157048), and H2020, EuroCPS, (GA 644090).

\section{REFERENCES}

[1] Billinghurst, Mark, and Daniela Busse. "Rapid Prototyping for Wearables: Concept Design and Development for head-and wristmounted Wearables (Smart Watches and Google Glass)." Proceedings of the Ninth International Conference on Tangible, Embedded, and Embodied Interaction. ACM, 2015.

[2] Gorlatova, M., Sarik, J., Grebla, G., Cong, M., Kymissis, I. and Zussman, G., 2014, June. Movers and shakers: Kinetic energy harvesting for the internet of things. In The 2014 ACM international conference on Measurement and modeling of computer systems (pp. 407-419). ACM.

[3] P. Mitcheson, E. Yeatman, G. Rao, A. Holmes, and T. Green. Energy harvesting from human and machine motion for wireless electronic devices. Proc. of the IEEE, 96(9):1457 -1486, Sept. 2008

[4] N. Shenck and J. Paradiso, "Energy scavenging with shoe-mounted piezoelectrics," IEEE micro, vol. 3, pp. 30-42, 2001.

[5] J. A. Paradiso and T. Starner, "Energy scavenging for mobile and wireless electronics," Pervasive Computing, vol. 4(1), pp. 18-27, 2005.

[6] R.-S. Liu, P. Sinha, and C. E. Koksal. Joint energy management and resource allocation in rechargeable sensor networks. In Proc. IEEE INFOCOM'10, Mar. 2010.

[7] Magno, M.; Porcarelli, D.; Brunelli, D.; Benini, L., "InfiniTime: A MultiSensor Energy Neutral Wearable Bracelet," Proceedings of the IEEE International Green Computing Conference, 2014.

[8] A. S. Weddell, M. Magno, G. V. Merrett, D. Brunelli, B. M. Al-Hashimi and L. Benini, "A Survey of Multi-Source Energy Harvesting Systems," in Design, Automation \& Test in Europe Conference \& Exhibition (DATE), 2013 , pp.905-910, March 2013

[9] Spadaro, L., Magno, M. and Benini, L., 2016, April. KinetiSee-A Perpetual Wearable Camera Acquisition System with a Kinetic Harvester. In 2016 15th ACM/IEEE International Conference on Information Processing in Sensor Networks (IPSN) (pp. 1-2). IEEE.

[10] Carli, D.; Brunelli, D.; Benini, L.; Ruggeri, M., "An effective multi-source energy harvester for low power applications," Design, Automation \& Test in Europe Conference \& Exhibition (DATE), 2011 , vol., no., pp.1,6, 1418 March 2011

[11] Srbinovski, B., Magno, M., Edwards-Murphy, F., Pakrashi, V. and Popovici, E., 2016. An Energy Aware Adaptive Sampling Algorithm for Energy Harvesting WSN with Energy Hungry Sensors. Sensors, 16(4), p. 448 .
[12] Bergonzini, C.; Brunelli, D.; Benini, L., "Algorithms for harvested energy prediction in batteryless wireless sensor networks," Advances in sensors and Interfaces, 2009. IWASI 2009. 3rd International Workshop on , vol., no., pp.144,149, 25-26 June 2009

[13] Mauriello, Matthew, Michael Gubbels, and Jon E. Froehlich. "Social fabric fitness: the design and evaluation of wearable E-textile displays to support group running." Proceedings of the 32nd annual ACM conference on Human factors in computing systems. ACM, 2014.

[14] Magno, M.; Boyle, D.; Brunelli, D.; O'Flynn, B.; Popovici, E.; Benini, L., "Extended Wireless Monitoring Through Intelligent Hybrid Energy Supply," Industrial Electronics, IEEE Transactions on, vol.61, no.4, pp.1871,1881, April 2014

[15] Brogan, Q.; O'Connor, T.; Dong Sam Ha, "Solar and thermal energy harvesting with a wearable jacket," Circuits and Systems (ISCAS), 2014 IEEE International Symposium on, vol., no., pp.1412,1415, 1-5 June 2014

[16] Wahbah, M.; Alhawari, M.; Mohammad, B.; Saleh, H.; Ismail, M., "Characterization of Human Body-Based Thermal and Vibration Energy Harvesting for Wearable Devices," Emerging and Selected Topics in Circuits and Systems, IEEE Journal on , vol.4, no.3, pp.354,363, Sept. 2014

[17] http://www.kinetron.eu/wp-content/uploads/2015/01/MGS-26.4.pdf

[18] Mitcheson, Paul D. "Energy harvesting for human wearable and implantable bio-sensors." Engineering in Medicine and Biology Society (EMBC), 2010 Annual International Conference of the IEEE. IEEE, 2010.

[19] Leonov, V., "Thermoelectric Energy Harvesting of Human Body Heat for Wearable Sensors,"Sensors Journal, IEEE, vol.13, no.6, pp.2284,2291, June 2013

[20] P. K. W. a. J. M. R. S. Roundy, "Energy scavenging for wireless sensor networks with special focus on vibrations," in Kluwer Academic Publishers, 2004.

[21] H. Hu, H. Xue and Y. Hu, "A spiral-shaped harvester with an improved harvesting element and an adaptive storage circuit," Ultrasonics, Ferroelectrics, and Frequency Control, vol. 54, no. 6, pp. 1177-1187, 2007.

[22] Y. Jeon, R. Sood, J. Jeong and S. Kim, "MEMS power generator with transverse mode thin film PZT," Sensors and Actuators A: Physical, vol. 122, no. 1, pp. 16-22, 2005.

[23] H. F. J.Q. Liu, Z. Xu, X. Mao, X. Shen, D. Chen, H. Lia and B. Cai, “A MEMS-based piezoelectric power generator array for vibration energy harvesting," Microelectronics Journal, vol. 39, no. 5, pp. 802-806, 2008.

[24] M. Renaud, K. Karakaya, T. Sterken, P. Fiorini, C. V. Hoof and R. Puers, "Fabrication, modelling and characterization of MEMS piezoelectric vibration harvesters," Sensors and Actuators, vol. A: Physical, no. 145, pp. 380-386, 2008. 